\title{
AN ELLIPTIC SYSTEM OF INTEGRAL EQUATIONS ON SUMMABLE FUNCTIONS*
}

BY

\section{J. H. BINNEY}

1. Introduction. Given $A(e)$ and $B(e)$ two additive functions of point sets $e$, measurable in the Borel sense and contained in a simply connected and bounded plane open region $T$, we shall consider the elliptic system of integral equations

$$
\begin{aligned}
\int_{0} \phi(x, y) d y+\theta(x, y) d x & =A(\sigma), \\
\int_{0}-\theta(x, y) d y+\phi(x, y) d x & =B(\sigma),
\end{aligned}
$$

in which $s$ is a (variable) simple closed rectifiable curve in $T$ and $\sigma$ its interior region. In one sense it would be more natural to employ, in the right hand members of equations (1), the functions of curves which "correspond" $\ddagger$ to $A(e)$ and $B(e)$, rather than the functions of point sets themselves, since the curvilinear integrals are functions of curves. But aside from the difficulty of defining additive functions of curves on a family of curves as general as rectifiable ones, it turns out that we need to consider only curves $s$ where the functions of point sets are regular, that is, such that

$$
A(\sigma)=A(\sigma+s), \quad B(\sigma)=B(\sigma+s) ;
$$

in fact, only curves such that if $e^{\prime \prime}$ is any set of points on $s$ itself measurable Borel on $s$, then $A\left(e^{\prime \prime}\right)=0$ and $B\left(e^{\prime \prime}\right)=0$.

In this paper, $\S$ a general solution of the pair of equations (1) is obtained, supposing merely that the functions $\phi$ and $\theta$ are summable superficially everywhere in $T$. This result is expressed in Theorem IV, below.

Let

* Presented to the Society, August 31, 1932; received by the editors May 31, 1934.

$\dagger$ "Additive" is understood as "completely additive," that is, additive over a denumerable infinity of distinct sets. An additive function is therefore bounded.

$\ddagger$ Evans, Fundamental points of potential theory, Rice Institute Pamphlet, vol. 7 (1920), pp. 252-329. See pp. 261 and 268.

$\S$ The author wishes to express his indebtedness for assistance in this problem to Professor G. C. Evans, who has discussed continuous solutions of the related pair of partial differential equations in An elliptic system related to Poisson's equation, Acta Szeged, vol. 6 (1932), pp. 27-33, and to Dr. A. J. Maria, who has also made suggestions on important points of the treatment. 


$$
\begin{aligned}
\phi_{0}(M) & \equiv \frac{1}{2 \pi} \int_{T} \frac{1}{M P}\left[\cos (M P, y) d B\left(e_{P}\right)-\cos (M P, x) d A\left(e_{P}\right)\right], \\
\theta_{0}(M) & \equiv \frac{1}{2 \pi} \int_{T} \frac{1}{M P}\left[\cos (M P, x) d B\left(e_{P}\right)+\cos (M P, y) d A\left(e_{P}\right)\right] .
\end{aligned}
$$

These functions are defined almost everywhere and are summable over the region $T{ }^{*}$ We shall see that these are particular solutions of the pair of equations (1).

2. Lemmas on convergence. It is necessary first to establish the following lemmas.

LEMMa A. If $f(e)$ is a non-negative additive function of point sets $e$ in $T$, measurable in the Borel sense, and $s$ is a simple closed rectifiable curve in $T$, on which $\int_{T}[1 /(M P)] d f\left(e_{P}\right)$ represents a summable junction of $M$ with respect to arc length, then $f\left(e^{\prime \prime}\right)=0$ for every set $e^{\prime \prime}$, composed of points of $s$ and measurable Borel on $s$, and the equation

$$
\int_{T} \frac{1}{M P} d f\left(e_{P}\right)=\int_{T-s} \frac{1}{M P} d f\left(e_{P}\right)
$$

is valid.

The lemma is of course equally true if $s$ is a simple rectifiable arc, and the proof which follows requires merely an obvious modification to cover this case.

At any point $M$ of $s$ for which $\int_{T}[1 /(M P)] d f\left(e_{P}\right)$ converges, we have

$$
\int_{T} \frac{1}{M P} d f\left(e_{P}\right)=\int_{T-s} \frac{1}{M P} d f\left(e_{P}^{\prime}\right)+\int_{s} \frac{1}{M P} d f\left(e_{P}{ }^{\prime \prime}\right)
$$

where $e^{\prime \prime}=e \cdot s$ and $e^{\prime}=e \cdot(T-s)$, that is, the parts of $e$ on $s$ and in $T-s$, respectively. These sets are measurable Borel if $e$ is measurable Borel and $f(e)=f\left(e^{\prime}\right)+f\left(e^{\prime \prime}\right)$ since $e=e^{\prime}+e^{\prime \prime}$ and $e^{\prime}$ and $e^{\prime \prime}$ have no common points. To show that (3) holds, we let

$$
\begin{aligned}
h_{n}(M, P) & =\frac{1}{M P}, & & M P>\frac{1}{n}, \\
& =n, & & M P \leqq \frac{1}{n} .
\end{aligned}
$$

This is a continuous function of $(M, P)$ and is such that $h_{n}(M, P)$

\footnotetext{
* Evans, Rice Institute Pamphlet, loc. cit. See p. 263.
} 
$\leqq h_{n+1}(M, P)$. Since $h_{n}(M, P)$ is uniformly continuous as a function of $P$ in the region $T-s$, in fact in the whole plane, we may show from the definition of the Stieltjes integral that

$$
\int_{T} h_{n}(M, P) d f\left(e_{P}\right)=\int_{T-\bullet} h_{n}(M, P) d f\left(e_{P}^{\prime}\right)+\int_{\bullet} h_{n}(M, P) d f\left(e_{P}^{\prime \prime}\right) .
$$

All three terms of this equation are increasing functions of $n$, and since the left hand member remains bounded as $n$ becomes infinite, both terms of the right hand member do also. Hence the resulting generalized integrals converge and yield the desired equation (3).

Hence if the left hand member of equation (3) represents a summable function of $M$ on the curve $s$, the same is true of the function

$$
\int_{0} \frac{1}{M P} d f\left(e_{P}^{\prime \prime}\right) \text {. }
$$

But, as is seen by direct calculation, this can be true only if $f\left(e^{\prime \prime}\right) \equiv 0$.

A well known property, which is deduced with the aid of Fubini's theorem on multiple integrals, is the following:

LEMma B. If $f(e)$ is an additive function of point sets $e$, measurable Borel in $T$, then the integral $\int_{T}[1 /(M P)] d f\left(e_{P}\right)$ exists for almost all $M$ in $T$ and is a summable function of $M$ on almost all rectangular contours in $T$, whose sides are parallel to two fixed rectangular directions $x$ and $y$.

When the integrals in (4) below are suitably defined we may prove the following lemma.

Lemma C. If $f(e)$ is an additive function of point sets $e$ in $T$, measurable Borel, and $s$ is a simple closed rectifiable curve in $T$, on which

$$
\int_{T} \frac{1}{M P}\left|d f\left(e_{P}\right)\right|
$$

represents a summable function of the point $M$ with respect to arc length, then the integrals

$$
\int_{T} \frac{1}{M P} \cos \left(M P, s_{M}\right) d f\left(e_{P}\right), \quad \int_{T} \frac{1}{M P} \cos \left(M P, n_{M}\right) d f\left(e_{P}\right)
$$

exist almost everywhere in $T$ as summable functions of $M$ with respect to arc length on $s$ and the equations

$$
\int_{0} d s_{M} \int_{T} \frac{1}{M P} \cos \left(M P, s_{M}\right) d f\left(e_{P}\right)
$$


(6)

$$
\begin{gathered}
=\int_{T-s} d f\left(e_{P}\right) \int_{s} \frac{1}{M P} \cos \left(M P, s_{M}\right) d s_{M}=0 \\
\int_{s} d s_{M} \int_{T} \frac{1}{M P} \cos \left(M P, n_{M}\right) d f\left(e_{P}\right) \\
=\int_{T-s} d f\left(e_{P}\right) \int_{s} \frac{1}{M P} \cos \left(M P, n_{M}\right) d s_{M}=2 \pi f(\sigma)
\end{gathered}
$$

are valid, where $\sigma$ is the set of points interior to s.

In this paper we take $n_{M}$ to mean the direction of the interior normal to the curve $s$ at the point $M$.

We notice that $\cos \left(M P, s_{M}\right), \cos \left(M P, n_{M}\right)$ are not defined when $P=M$ or when $M$ is a point on the curve $s$ where the direction of $s$ is not definite. Evidently, however, we may define them in these exceptional cases so that the functions are measurable Borel in the closed space $R_{3}$ in which coordinates are $x=x_{P} ; y=y_{P} ; z=s_{M}, s_{M}$ being measured from some fixed point on $s$ and varying over the length of $s$, and $P \equiv(x, y)$ being confined to a closed rectangle $R$ which includes the region $T$. We extend the definition of $f(e)$ throughout $R$ by writing $f(e)=f(e \cdot T)$.

Since the integral over $T$ with respect to $f(e)$ is the same as the integral over $T-s$, and since the points $M$ on $s$ where $s$ has no tangent direction form a set of measure zero on $s$, independent of $P$ in $T-s$, the values of the iterated integrals in (5) and (6) are independent of the definitions assigned to these cosines at the exceptional points. The lemma is then verified by a change in the order of integration which is justified by the existence of the integral*

$$
\int_{s} d s_{M} \int_{r_{-s}} \frac{1}{M P}\left|d f\left(e_{P}\right)\right| .
$$

A lemma of slightly different type is the following:

Lemma D. Let $\alpha, x$ represent any two fixed directions. Then, with the hypotheses on s and $f(e)$ of Lemma $\mathrm{C}$, the integral

$$
\int_{0} d x \int_{T} \frac{1}{M P} \cos (M P, \alpha) d f\left(e_{P}\right)=\int_{.} d x \int_{T_{-s}} \frac{1}{M P} \cos (M P, \alpha) d f\left(e_{P}\right)
$$

exists, and is equal to

$$
\int_{T-8} d f\left(e_{P}\right) \int_{\delta} \frac{1}{M P} \cos (M P, \alpha) d x .
$$

* G. C. Evans, Rice Institute Pamphlet, loc. cit., p. 258. 
The integral in Lemma D is an iteration of two Daniell $S$-integrals. ${ }^{*}$ For its treatment we make use of the following proposition:

LEMMA E. If $f(t)$ is measurable Borel and if $x(t)$ is absolutely continuous in $t$ such that $f(t) x^{\prime}(t)=f(t) d x / d t$ is summable over $(a, b)$, then $\int_{a}^{b} f(t) d x(t)$ exists and

$$
\int_{a}^{b} f(t) d x(t)=\int_{a}^{b} f(t) x^{\prime}(t) d t .
$$

We note that if $f(t)$ is summable and $x^{\prime}(t)$ is bounded, $f(t) x^{\prime}(t)$ is summable over $(a, b)$.

We first prove the lemma for $x(t)$ monotonic-increasing. If $f(t)$ is continuous both members of the above equation exist and they are equal. With $x^{\prime}(t) \geqq 0$, both members are general $I$-integrals of $f(t)$ (a Lebesgue integral is merely a special case of such an integral). Hence if the right hand member exists, the left member does also, and the equality is unchanged. But the right member exists if $f(t) x^{\prime}(t)$ is summable.

If $x(t)$ is no longer monotonic-increasing, from the measurability of $f(t), x^{\prime}(t)$ and the summability of $f(t) \cdot x^{\prime}(t)$ follows the summability of the functions

$$
f(t) \cdot \frac{1}{2}\left\{\left|x^{\prime}(t)\right| \pm x^{\prime}(t)\right\} .
$$

Hence if we denote by $x^{+}(t), x^{-}(t)$ the positive and negative variation functions respectively for $x(t)$, with derivatives

$$
x^{+\prime}(t)=\frac{1}{2}\left\{\left|x^{\prime}(t)\right|+x^{\prime}(t)\right\}, \quad x^{-\prime}(t)=\frac{1}{2}\left\{\left|x^{\prime}(t)\right|-x^{\prime}(t)\right\},
$$

the I-integrals $\int_{a}^{b} f(t) d x^{+}(t)=\int_{a}^{b} f(t) x^{+^{\prime}}(t) d t$ and $\int_{a}^{b} f(t) d x^{-}(t)=\int_{a}^{b} f(t) x^{-1}(t) d t$ both exist, and $\int_{a}^{b} f(t) d x(t)$, which is an $S$-integral and the difference of two $I$-integrals, exists and has the assigned value. $\dagger$

Consider now any function $Q(M)$ summable with respect to arc length $s_{M}$ on the simple closed rectifiable curve $s$. Let us write $x_{M}=x\left(s_{M}\right)$ to represent the projection of the $\operatorname{arc} R M=s_{M}$ on $O X$, where $R$ is some fixed point of the curve $s$. Then $x\left(s_{M}\right)$ is an absolutely continuous function of arc length $s_{M}$. In fact,

$$
x_{M}=x\left(s_{M}\right)=\int_{s_{M}} \cos \left(x, s_{M}\right) d s_{M} .
$$

Hence the derivative $d x / d s_{M}=\cos \left(x, s_{M}\right)$ exists for almost all points $M$ on $s$ and is bounded in absolute value by unity. Hence by Lemma $\mathrm{E}$,

* P. J. Daniell, A general form of integral, Annals of Mathematics, vol. 19 (1918), pp. 279-294.

† P. J. Daniell, loc. cit. 


$$
\int_{.} Q(M) d x_{M} \text { exists and }=\int_{0} Q(M) \cos \left(x, s_{M}\right) d s_{M} .
$$

We return now to the proof of Lemma $D$. Since the function

$$
\int_{T} \frac{1}{M P}\left|d f\left(e_{P}\right)\right|
$$

is summable with respect to $s_{M}$ on $s$ and since it dominates the measurable function

$$
\int_{T} \frac{1}{M P} \cos (M P, \alpha) d f\left(e_{P}\right),
$$

then the latter is summable with respect to $s_{M}$. Hence we may take

$$
Q(M)=\int_{T} \frac{1}{M P} \cos (M P, \alpha) d f\left(e_{P}\right) .
$$

Therefore

$$
\begin{aligned}
\int_{0} d x_{M} \int_{T} \frac{1}{M P} \cos (M P, \alpha) d f\left(e_{P}\right) \\
=\int_{s} d s_{M} \int_{T} \frac{1}{M P} \cos (M P, \alpha) \cos \left(x, s_{M}\right) d f\left(e_{P}\right) .
\end{aligned}
$$

But now the change of order of integration may be justified in the same manner as in Lemma C. Also $f(e \cdot s)=0$ and our integral is therefore

$$
\int_{T-\_} d f\left(e_{P}\right) \int_{0} \frac{1}{M P} \cos (M P, \alpha) \cos \left(x, s_{M}\right) d s_{M}
$$

and this again is

$$
\int_{T \rightarrow} d f\left(e_{P}\right) \int_{s} \frac{1}{M P} \cos (M P, \alpha) d x_{M} .
$$

This completes the proof of Lemma $D$.

3. The general solution of equations (1). We are now in a position to prove the following theorem.

THEOREM I. The functions $\phi_{0}$ and $\theta_{0}$ given by (2) satisfy the pair of equations (1) on all simple closed rectifiable curves $s$ in $T$ on which

$$
\int_{T} \frac{1}{M P}\left|d A\left(e_{P}\right)\right|, \quad \int_{T} \frac{1}{M P}\left|d B\left(e_{P}\right)\right|
$$

represent summable functions of $M$ with respect to arc length on $s$. 
Since the functions

$$
\int_{T} \frac{1}{M P}\left|d A\left(e_{P}\right)\right|, \quad \int_{T} \frac{1}{M P}\left|d B\left(e_{P}\right)\right|
$$

are summable with respect to arc length on $s$ and since $x$ and $y$ are absolutely continuous functions of $s_{M}$ with bounded derivatives, we can conclude from Lemma $\mathrm{E}$, as in Lemma $\mathrm{D}$, that the integral

$$
\int_{0} \phi_{0} d y+\theta_{0} d x
$$

exists and, moreover, that

$$
\int_{.} \phi_{0} d y+\theta_{0} d x=\int\left\{\phi_{0} \frac{d y}{d s_{M}}+\theta_{0} \frac{d x}{d s_{M}}\right\} d s_{M} .
$$

Hence we are justified in substituting $\phi_{0}$ and $\theta_{0}$ in the left hand member of the first of the equations (1). Doing this and noting that, almost everywhere on $s$,

we get

$$
\begin{aligned}
& \frac{d y}{d s_{M}}=\cos \left(y, s_{M}\right)=-\cos \left(x, n_{M}\right), \\
& \frac{d x}{d s_{M}}=\cos \left(x, s_{M}\right)=\cos \left(y, n_{M}\right),
\end{aligned}
$$

$$
\int_{0} \phi_{0} d y+\theta_{0} d x=\int_{0}\left\{-\phi_{0} \cos \left(x, n_{M}\right)+\theta_{0} \cos \left(y, n_{M}\right)\right\} d s_{M} .
$$

But the right hand member may be written as follows, since $A(e \cdot s) \equiv 0$ $\equiv B(e \cdot s)$ :

$$
\begin{aligned}
\frac{1}{2 \pi} \int_{0} d s_{M} & \int_{T_{-}} \frac{1}{M P}\left\{-\cos (M P, y) \cos \left(x, n_{M}\right) d B\left(e_{P}\right)\right. \\
& +\cos (M P, x) \cos \left(x, n_{M}\right) d A\left(e_{P}\right)+\cos (M P, x) \cos \left(y, n_{M}\right) d B\left(e_{P}\right) \\
& \left.+\cos (M P, y) \cos \left(y, n_{M}\right) d A\left(e_{P}\right)\right\} \\
=\frac{1}{2 \pi} & \int_{0} d s_{M} \int_{T-s} \frac{1}{M P}\left\{\left[\cos (M P, x) \cos \left(y, n_{M}\right)\right.\right. \\
& \left.-\cos (M P, y) \cos \left(x, n_{M}\right)\right] d B\left(e_{P}\right)+\left[\cos (M P, x) \cos \left(x, n_{M}\right)\right. \\
& \left.\left.+\cos (M P, y) \cos \left(y, n_{M}\right)\right] d A\left(e_{P}\right)\right\}
\end{aligned}
$$




$$
\begin{aligned}
& =\frac{1}{2 \pi} \int_{.} d s_{M} \int_{T_{-\varepsilon}} \frac{1}{M P}\left\{\left[\cos (M P, x) \cos \left(x, s_{M}\right)\right.\right. \\
& \left.\quad+\cos (M P, y) \cos \left(y, s_{M}\right)\right] d B\left(e_{P}\right)+\left[\cos (M P, x) \cos \left(x, n_{M}\right)\right. \\
& \left.\left.\quad+\cos (M P, y) \cos \left(y, n_{M}\right)\right] d A\left(e_{P}\right)\right\} .
\end{aligned}
$$

We have, for almost all $M$ on $s$,

$$
\begin{aligned}
& \cos (M P, x) \cos \left(x, n_{M}\right)+\cos (M P, y) \cos \left(y, n_{M}\right)=\cos \left(M P, n_{M}\right), \\
& \cos (M P, x) \cos \left(x, s_{M}\right)+\cos (M P, y) \cos \left(y, s_{M}\right)=\cos \left(M P, s_{M}\right) .
\end{aligned}
$$

Hence for almost all $M$ on $s$, the inside integrals take the desired form

$$
\int_{T-s} \frac{1}{M P} \cos \left(M P, s_{M}\right) d B\left(e_{P}\right), \quad \int_{T_{-s}} \frac{1}{M P} \cos \left(M P, n_{M}\right) d A\left(e_{P}\right) .
$$

Hence the Lebesgue integral with respect to $s_{M}$ of the inside integrals has the value

$$
\begin{aligned}
& \frac{1}{2 \pi} \int_{\delta} d s_{M} \int_{T_{-s}} \frac{1}{M P} \cos \left(M P, s_{M}\right) d B\left(e_{P}\right) \\
& \quad+\frac{1}{2 \pi} \int_{s} d s_{M} \int_{T_{-s}} \frac{1}{M P} \cos \left(M P, n_{M}\right) d A\left(e_{P}\right) .
\end{aligned}
$$

By making use now of the results of Lemma $\mathrm{C}$ we see that

$$
\int_{0} \phi_{0} d y+\theta_{0} d x=A(\sigma)
$$

and $\phi_{0}, \theta_{0}$ satisfy the first equation of (1). In a similar manner we can show that $\phi_{0}$ and $\theta_{0}$ also satisfy the second equation of (1) for $s$.

From Lemma B and Theorem I, we have the following corollary.

Corollary. The functions $\phi_{0}$ and $\theta_{0}$ given by (2) satisfy the pair of equations (1) on almost all rectangles in $T$, with sides parallel to $x$ and $y$.

These results lead immediately to the following theorem:

THEOREM II. If the functions $\bar{\phi}$ and $\bar{\theta}$ are solutions of equations (1) on almost all rectangles in $T$, the functions

$$
\phi=\bar{\phi}-\phi_{0}, \quad \theta=\bar{\theta}-\theta_{0}
$$

satisfy the pair of equations

$$
\begin{array}{r}
\int_{s} \phi d y+\theta d x=0, \\
\int_{8}-\theta d y+\phi d x=0,
\end{array}
$$

on almost all rectangles in $T, \phi_{0}$ and $\theta_{0}$ being given by (2). 
We state the following theorem.*

THEOREM III. If $\phi$ and $\theta$ are two functions summable superficially over every closed region interior to $T$ and satisfy equations (7) on almost all rectangles in $T$, then there exists a function $\Psi(M)$ harmonic in $T$, such that

$$
\frac{\partial \Psi}{\partial x}=\theta(M), \quad \frac{\partial \Psi}{\partial y}=\phi(M)
$$

for almost all $M$ in $T$.

Finally, we may summarize the results of the preceding pages in the following theorem:

TheOREM IV. The functions

$$
\phi \equiv \phi_{0}+\frac{\partial \psi}{\partial y} ; \quad \theta \equiv \theta_{0}+\frac{\partial \psi}{\partial x}
$$

where $\phi_{0}$ and $\theta_{0}$ are given by (2) and where $\psi$ is an arbitrary solution of Laplace's equation in $T$, form a system of solutions of the pair of equations (1) for all simple closed rectifiable curves $s$, interior to the region $T$, on which the integrals

$$
\int_{T} \frac{1}{M P}\left|d A\left(e_{P}\right)\right|, \quad \int_{T} \frac{1}{M P}\left|d B\left(e_{P}\right)\right|
$$

represent summable functions of $M$ on $s$, with respect to $d s_{M}$; in particular the functions (8) are solutions of (1) for almost all rectangular contours interior to $T$ with sides parallel to given directions $x$ and $y$.

Conversely, if $\phi$ and $\theta$ are summable superficially over any closed region interior to $T$ and are solutions of the pair of equations (1) on almost all rectangles, interior to the region $T$, with sides parallel to the axes, they may be expressed in the form (8) in $T-E(x, y)$, where $E(x, y)$ is a set of points of $T$ having superficial measure zero at most.

4. The complex plane. It is interesting to note that we might have begun our study of the pair of equations (1) by considering a single equation in complex variables.

* The editors of these Transactions have brought to the author's attention the fact that substantially this theorem is proved by V. S. Fedoroff, Sur le theorème de Morera, Moscow Mathematicheskii Sbornik, vol. 40 (1933), pp. 168-179. Consequently it is unnecessary to give a proof of Theorem III. See also G. C. Evans, Note on a theorem of Bocher, American Journal of Mathematics, vol. 50 (1928), pp. 123-126. For Theorem III a function $\Psi$ is constructed which is a "potential of its generalized derivatives" $\theta, \phi$, and satisfies the second of $(7) ; \Psi$ is then harmonic by the generalized theorem of Bôcher. 
In order to show the connection of this pair of equations with a single equation in the complex plane, we let $f(z)$ be a function of the complex variable $z=x+i y$ such that its real and its imaginary parts are summable superficially over any closed region which is interior to a simply connected plane bounded open region $T$. Let $\Phi(e)$ be a completely additive complex-valued function of point sets $e$ in $T$ measurable in the Borel sense. We now consider the equation

$$
\int_{\varepsilon} f(z) d z=\Phi(\sigma)
$$

where $s$ is a simple closed rectifiable curve in the region $T$ and $\sigma$ is the set of interior points of $s$. If we place

$$
\Phi(e)=B(e)+i A(e)
$$

and let

$$
f(z)=\phi(x, y)+i \theta(x, y)
$$

equation (9) becomes

$$
\int_{s}(\phi+i \theta)(d x+i d y)=B(\sigma)+i A(\sigma)
$$

If we now equate the real and the imaginary parts we obtain the pair of equations

$$
\begin{array}{r}
\int_{s} \phi d y+\theta d x=A(\sigma), \\
\int_{s}-\theta d y+\phi d x=B(\sigma)
\end{array}
$$

which we have been studying. We may accordingly state the following theorem:

THEOREM V. If $s$ is a simple closed rectifiable curve in $T$ on which the integrals

$$
\int_{T} \frac{1}{M P}\left|d A\left(e_{P}\right)\right|, \quad \int_{T} \frac{1}{M P}\left|d B\left(e_{P}\right)\right|
$$

represent summable functions of $M$ with respect to arc length $s_{M}$, then

$$
f(z) \equiv F(z)+\frac{1}{2 \pi} \int_{T} \frac{1}{M P} e^{i \alpha} d \Phi\left(e_{P}\right)
$$


is a solution of equation (9), where $\alpha$ is the angle $(M P, y)$ and $F(z)$ is an arbitrary holomorphic function in $T$.

Since

$$
\int_{0} F(z) d z=0
$$

by the Cauchy integral theorem, it only remains to show that

$$
\frac{1}{2 \pi} \int_{T} \frac{1}{M P} e^{i \alpha} d \Phi\left(e_{P}\right)
$$

satisfies equation (9). And this is verified by direct calculation.

As a result of this theorem and of Lemma $B$ we have the following corollary.

Corollary. The function

$$
f(z) \equiv F(z)+\frac{1}{2 \pi} \int_{T} \frac{1}{M P} e^{i \alpha} d \Phi\left(e_{P}\right)
$$

is a solution of equation (9) on almost all rectangles in $T$.

We also have a converse result expressed by the following theorem.

THEOREM VI. If $\phi$ and $\theta$ are two functions summable over every closed region interior to $T$ and if $\phi+i \theta$ is a solution of equation (9) on almost all rectangular contours $s$ in $T$ whose sides are parallel to the given directions $x$ and $y$, then $\phi+i \theta$ may be expressed in the form (10) in $T-E(x, y)$ where $E(x, y)$ is a set of points of $T$ of superficial measure zero.

Since $\phi+i \theta$ is a solution of the equation (9) on almost all rectangles $s$ in $T$ we have

$$
\int_{.}(\phi+i \theta) d z=\Phi(\sigma)
$$

In fact the functions

$$
u=\phi-\phi_{0}, \quad v=\theta-\theta_{0}
$$

satisfy the equations

$$
\int_{s} u d y+v d x=0, \quad \int_{0}-v d y+u d x=0
$$


on almost all rectangles in $T$. Hence, almost everywhere in $T$,

$$
u=\frac{\partial \Psi}{\partial y}, \quad v=\frac{\partial \Psi}{\partial x},
$$

where $\Psi$ is harmonic in $T$. But then the function

$$
F(z)=u+i v=\frac{\partial \Psi}{\partial y}+i \frac{\partial \Psi}{\partial x}
$$

is holomorphic in $T$.

Rice InstrTute,

Houston, Texas 